\title{
Hydrodynamical Phenomena at Preparation of Earthquake
}

\author{
Igor Petrovich Dobrovolsky \\ Institute of Physics of the Earth, Russian Academy of Sciences, Moscow, Russia \\ Email: dipedip@gmail.com
}

Received 7 July 2014; revised 10 August 2014; accepted 12 September 2014

Copyright (C) 2014 by author and OALib.

This work is licensed under the Creative Commons Attribution International License (CC BY).

http://creativecommons.org/licenses/by/4.0/

(c) (i) Open Access

\begin{abstract}
Variations in level of fluid in wells caused by Earth tides and earthquakes are governed by two leading processes: deformation of medium and filtration. These processes are described by identical systems of equations and are united by the term "hydrodynamical phenomena". The exact solution is obtained for the problem of level variations in wells due to Earth tides. The similar problem is solved for short-term precursors of a tectonic earthquake. Results are consistent with data of field observations.
\end{abstract}

\section{Keywords}

Earth Tides, Short-Term Precursors of Earthquakes, Level of Liquid in a Well

Subject Areas: Fluid Mechanics, Geology

\section{Introduction}

Often before an earthquake (during a period of short-term precursors) there are variations in level of fluid in wells and boreholes, dimness of water in wells, variations in fluid temperature and disturbances in the regime of natural fountains. If they are really caused by an earthquake preparation all these phenomena are defined as hydrodynamical earthquake precursors.

Short-term precursors, the earthquake itself and its aftershocks are the processes of the same physical sense which enables their identical phenomenological description and calculation. We comment this note. The cycle of the tectonic earthquake separates into two different stages: accumulation of energy and its release. Phenomena accompanying the stage accumulation of energy are defined as long-term precursors of an earthquake. This stage is conditioned by the appearance and development of the heterogeneity in the bowels of the Earth [1]. After that the disintegration of the heterogeneity begins and the release of energy stored. This stage consists of three phases: relatively short phase of short-term precursors, almost instantaneous act of earthquake's focus and the prolonged aftershocks activity. Thus these phases are physically identical in the sense that they change the elastic 
potential energy of the medium in the same direction. If the state of the medium after the period of long-term precursors is treated as initial position then the following behavior displays three decreases of elastic potential energy.

Action of the hydrodynamical phenomena due to an earthquake depends on the properties of the rocks in the vicinity of a well (borehole) and its location with respect to the hypocenter of the earthquake. However the first mechanism also characterizes the variations of level in wells due of the Earth tides. Therefore we can discuss hydrodynamical phenomena in general and describe them by identical equations.

Hydrodynamical precursors are known for a long time and their description is usually included into monographs (for example [2]). A noticeable paper in this field is [3] that contains the plots of variations of water level by $15 \mathrm{~cm}$ before the Przhevalsk earthquake of $1970(M=6.8)$ at an epicentral distance of $30 \mathrm{~km}$. But there are also other examples. The striking example of the precursory anomaly is given in [4]: groundwater level decreased by $57 \mathrm{~m}$ before the earthquake of magnitude 5.4 at epicentral distance of $70 \mathrm{~km}$. In the second case the epicentral distance is more, earthquake's magnitude is less but effect in 380 times more.

Seismologists undoubtedly accept the existence of hydrodynamical precursors; however, their quantitative characteristics are unclear. The reliable data about the distribution of hydrodynamical precursors in time and space are absent. Geophysicists are not able to calculate of hydrodynamical precursors.

The aim of the present paper is to calculate hydrodynamical phenomena: Earth's tidal and precursory variations in level of liquid in wells (boreholes) using identical systems of equations and to clarify data on distribution of hydrodynamical precursors on the Earth’s surface.

\section{The Equations of Two-Phase Medium}

We analyze the problem of filtration of fluids in porous two-phase medium. In the linear approximation, in the case of slow processes, when inertial forces and compressibility of the solid phase can be neglected with respect to the compressibility of fluid, the system of equations of dynamics of a porous liquid has the form [1]

$$
\begin{aligned}
& \operatorname{grad} P+\frac{\mu m_{0}}{k}(\mathbf{V}-\mathbf{U})=0, \\
& \frac{1}{m_{0}} \frac{\partial m}{\partial t}+\beta \frac{\partial P}{\partial t}+\operatorname{div} \mathbf{V}=0 .
\end{aligned}
$$

where $P(x, y, z, t)$ is the abundant porous pressure, $\mathbf{V}$ and $\mathbf{U}$ are velocities of fluid and solid phases, respectively.

The first equation of system (1) is Darsy law, the second equation reflects deformation of the fluid phase in elementary volume of a two-phase medium.

Transform the first term in the second equation of system (1). We consider representative volume $v_{0}$ of a medium large enough with respect to the sizes of pores, within which pores take the volume $v_{m_{0}}$. Then the current porosity $m$ is expressed by the formula

$$
m=\frac{v_{m_{0}}\left(1+e_{m}\right)}{v_{0}(1+e)}=m_{0} \frac{1+e_{m}}{1+e} .
$$

Obviously, $e$ and $e_{m}$ are connected among themselves and we write this relationship in the form $e_{m}=q e$. The value of $q$ depends on geometry of pores, stress state, properties of solid phase and properties of fluid. Note that the compressibility of fluid is higher than that of rocks in the Earth's crust by an order of magnitude. Calculations give the value $q=2$ in the case of spherical pores with water at all-round compression and porosity smaller than $20 \%$, when pores can be regarded as almost noninteracting. When the rocks contain an infinite water layer (fissure or disk-tape pore), and the system is compressed in the direction normal to the plane of the layer, calculations give $q=7-12$ for the porosity in the range of $10 \%-3 \%$. These results are easy to receive by solution of simple problems of the elasticity theory for a spherical pore and a fluid layer in an unlimited elastic medium. In the granulated medium of the type of sands, the total variation volume takes place due to redistribution of practically solid grains, and simple calculations give $q=1 / m_{0}$. Usually these media has a high porosity reaching $40 \%$ in the; for dry sands at $m_{0}=0.2, q=5$. Thereby, we can accept $q$ in the range $q=5-8$. 
Using expression (2), where we set $e_{m}=q e, q=$ const and $e=e(t)$, we obtain

$$
\frac{1}{m_{0}} \frac{\partial m}{\partial t}=\frac{q-1}{(1+e)^{2}} \frac{\partial e}{\partial t} \approx(q-1) \frac{\partial e}{\partial t} .
$$

here, $e$ is neglected with respect to 1 , because $e \leq 10^{-4}$.

Now system (1) takes form

$$
\begin{aligned}
& \operatorname{grad} P+\frac{\mu m_{0}}{k}(\mathbf{V}-\mathbf{U})=0, \\
& \beta \frac{\partial P}{\partial t}+\operatorname{div} \mathbf{V}=-(q-1) \frac{\partial e}{\partial t} .
\end{aligned}
$$

Excluding $\mathbf{V}$ from (4), we obtain the equation for $P$

$$
\operatorname{div} \frac{k}{\mu m_{0}} \operatorname{grad} P-\beta \frac{\partial P}{\partial t}=(q-1) \frac{\partial e}{\partial t}+\operatorname{div} \mathbf{U} .
$$

As $\mathbf{U}$ is velocity of the solid phase, then $\operatorname{div} \mathbf{U}=\partial e / \partial t$. Substituting this expression into (5), we finally obtain

$$
\operatorname{div} \frac{k}{\mu m_{0}} \operatorname{grad} P-\beta \frac{\partial P}{\partial t}=q \frac{\partial e}{\partial t} .
$$

When $k / \mu m_{0}=$ const, Equation (6) becomes the classic equation of diffusion with constant coefficients

$$
\frac{k}{\mu m_{0}} \Delta P-\beta \frac{\partial P}{\partial t}=q \frac{\partial e}{\partial t} .
$$

\section{General Statement of the Problem}

The Earth is modeled as a half-space. We consider an infinite thin horizontal water-saturated layer located at a depth within impenetrable rocks. The water-saturated layer is thin and all parameters and processes are homogeneous within it with respect to its thickness and, therefore, the filtration problem is two-dimensional. A well (borehole) is located in this layer and the liquid is at initial level. We should calculate deviations from this initial state and these deviations can be of any sign.

From Equation (7), equation for the porous pressure $P(r, \varphi, t)$ in a homogeneous medium $\left(\beta, \mu, q, k, m_{0}\right.$ are constant) is

$$
\frac{\partial P}{\partial t}-\kappa \Delta P=-\frac{q}{\beta} \frac{\partial e}{\partial t},
$$

where $\kappa=k / \mu \beta m_{0}$.

The right-hand side of this equation contains the volume strain $e$. By the natural definition the tensile deformation is positive and the compressible deformation is negative. It is necessary to mean it while solving the problem.

The first boundary condition is the condition in the well. The pressure variations in well are determined by the flow of liquid from the layer. By the Darsy law the condition takes the form

$$
\left.\left(P-\frac{\kappa \chi}{r} \int_{0}^{t} \frac{\partial P}{\partial r} \mathrm{~d} t^{\prime}\right)\right|_{r=r_{0}}=0,
$$

where $\chi=2 \sigma g h \beta m_{0}=2 \sigma g h k / \mu \kappa$.

The expression obtained is rather curious: it contains the dependence on angle $\varphi$. Borehole is as though divided into infinite number of sectors. The procedure of averaging over an angle $\varphi$ eliminates this shortcoming and the boundary condition becomes real

$$
\left.\left(\bar{P}-\frac{\kappa \chi}{r} \int_{0}^{t} \frac{\partial \bar{P}}{\partial r} \mathrm{~d} t^{\prime}\right)\right|_{r=r_{0}}=0,
$$


where the line denotes the averaging over an angle $\bar{P}=\frac{1}{2 \pi} \int_{0}^{2 \pi} P \mathrm{~d} \varphi$.

The second boundary condition (boundary condition at infinity) is $P(\infty, \varphi, t)=F$. The form of function $F$ depends on the certain problem but the general recipe of its definition is following. If $P^{\prime}(r, \varphi, t)$ is the solution of Equation (8) for the continuous layer (without a well) then $F=P^{\prime}(\infty, \varphi, t)$.

The differential Equation (8), the boundary condition (10), the boundary condition at infinity and the zero initial condition create the system for determination of function $P$ and pressure in the well $P_{w}(t)$

$$
\begin{aligned}
& \frac{\partial P}{\partial t}-\kappa\left(\frac{\partial^{2} P}{\partial r^{2}}+\frac{1}{r} \frac{\partial P}{\partial r}+\frac{1}{r^{2}} \frac{\partial^{2} P}{\partial \varphi^{2}}\right)=-\frac{q}{\beta} \frac{\partial e}{\partial t},\left.\quad\left(\bar{P}-\frac{\kappa \chi}{r} \int_{0}^{t} \frac{\partial \bar{P}}{\partial r} \mathrm{~d} t^{\prime}\right)\right|_{r=r_{0}}=0, \\
& P(\infty, \varphi, t)=F, \quad P(r, \varphi, 0)=0, \quad P_{w}(t)=\bar{P}\left(r_{0}, t\right) .
\end{aligned}
$$

\section{Tidal Variations of Level}

In the case of the Earth tides the investigated problem is the typical problem without initial conditions. We put $e=A \sin \omega t$. Then the problem becomes axisymmetric. If we will introduce dimensionless variables using expressions

$$
p=-\frac{\beta}{q A} P, \quad \rho=r \sqrt{\frac{\omega}{\kappa}}, \quad \tau=\omega t
$$

then from (11) we obtain the following system

$$
\frac{\partial p}{\partial \tau}-\left(\frac{\partial^{2} p}{\partial \rho^{2}}+\frac{1}{\rho} \frac{\partial p}{\partial \rho}\right)=\frac{\mathrm{d} \sin \tau}{\mathrm{d} \tau},\left.\quad\left(\frac{\partial p}{\partial \tau}-\frac{\chi}{\rho} \frac{\partial p}{\partial \rho}\right)\right|_{\rho=\rho_{0}}=0, \quad p(\infty, \tau)=\sin \tau .
$$

Solution of (13) is based on the fact that the homogeneous equation

$$
\frac{\partial u}{\partial \tau}-\left(\frac{\partial^{2} u}{\partial \rho^{2}}+\frac{1}{\rho} \frac{\partial u}{\partial \rho}\right)=0
$$

has the family of solutions via Kelvin functions with arbitrary constants

$$
u(\rho, \tau)=C_{1}(\operatorname{bei}(\rho) \cos (\tau-\alpha)+\operatorname{ber}(\rho) \sin (\tau-\alpha))+C_{2}(\operatorname{kei}(\rho) \cos (\tau-\alpha)+\operatorname{ker}(\rho) \sin (\tau-\alpha)) \text {. }
$$

To obtain this solution we search the solution in form $u=f(\rho) e^{i(\tau-\alpha)}$; then in the solution we go to Kelvin functions and determine the real and imaginary parts. Each of them is the solution of initial equation (we use the imaginary part). The solution equal to zero at infinity has the form

$$
u(\rho, \tau)=C(\operatorname{kei}(\rho) \cos (\tau-\alpha)+\operatorname{ker}(\rho) \sin (\tau-\alpha)) .
$$

Together with the particular solution we obtain the function

$$
p(\rho, \tau)=\sin \tau+C(\operatorname{kei}(\rho) \cos (\tau-\alpha)+\operatorname{ker}(\rho) \sin (\tau-\alpha)) .
$$

$C$ and $\alpha$ are determine from the first boundary condition in (13) (the second boundary condition is fulfilled for any $C$ and $\alpha$ ). The final result is following. Dimensionless pressure in the well is

$$
p_{w}(\tau)=p\left(\rho_{0}, \tau\right)=A_{w} \sin (\tau-\gamma),
$$

where

$$
\begin{gathered}
A_{w}=\frac{\chi B}{G}, \quad \cos \gamma=\frac{B^{2} \chi-\rho_{0} F}{B G}, G=\sqrt{B^{2} \chi^{2}-2 \rho_{0} F \chi+\rho_{0}^{2} S}, B=\sqrt{\operatorname{kei}_{1}^{2}\left(\rho_{0}\right)+\operatorname{ker}_{1}^{2}\left(\rho_{0}\right)}, \\
S=\operatorname{kei}^{2}\left(\rho_{0}\right)+\operatorname{ker}^{2}\left(\rho_{0}\right), \quad F=\operatorname{ker}\left(\rho_{0}\right) \frac{\operatorname{dkei}\left(\rho_{0}\right)}{\mathrm{d} \rho_{0}}-\operatorname{kei}\left(\rho_{0}\right) \frac{\operatorname{dker}\left(\rho_{0}\right)}{\mathrm{d} \rho_{0}} .
\end{gathered}
$$


Parameter $\gamma$ is the phase difference between tidal strains and level variations. To return to dimensional values we use expressions (12) and level variation is $l(t)=P_{w}(t) / \sigma g$. Such simple solution enables easy construction of the synthetic function of tidal variations in level by summing variations at various frequencies.

Let's make the short analysis of the solution (18). At $\rho_{0} \rightarrow 0$ we obtain the physically obvious result, i.e. $p_{w} \rightarrow \sin \tau$. Therefore the level amplitude is limited. Calculations also show that the phase difference $(\gamma)$ depending on frequency has the maximum but the decrease in $\gamma$ after it appears very weak. To calculate the amplitude value of level variation we assume: $A=10^{-7}$ (amplitude of the volume strain of the Earth tide), $q=7$, $r_{0}=0.5 \mathrm{~m}, \quad \kappa=25 / 864 \mathrm{~m}^{2} / \mathrm{s}, \quad \chi=3 \times 10^{-5}, \beta=4 \times 10^{-1} \mathrm{~Pa}^{-1}$. (In general $\kappa$ and $\chi$ should be defined from empirical data.) Then the level variation amplitude is equal to $1.2 \mathrm{~mm}$ and the amplitude limit is $175 \mathrm{~mm}$.

\section{Precursory Variations of Level}

The problem of calculation of precursory level variations is more complicated as compared to the previous one. The process is not oscillatory, the natural time scale is absent, initial condition is essentially zero and the boundary condition at infinity is also zero. The right-hand side of the differential equation depends on three variables (time, radius and angle) and involves two spatial scales: the local coordinate system where the filtration process occurs in the well vicinities and the global system of deformation of the Earth's crust due to an earthquake.

We represent the solution of system (11) as the Fourier row by $\varphi$

$$
P(r, \varphi, t)=W(r, t)+\sum_{n \neq 0} W_{n}(r, t) e^{i n \varphi} .
$$

After averaging expression (19) on angle $\varphi$ we receive $W(r, t)=\bar{P}$ and $P_{w}=W\left(r_{0}, t\right)$. As a result system (11) takes the form

$$
\begin{aligned}
& \frac{\partial W}{\partial t}-\kappa\left(\frac{\partial^{2} W}{\partial r^{2}}+\frac{1}{r} \frac{\partial W}{\partial r}\right)=-\frac{q}{\beta} \frac{\partial \bar{e}}{\partial t},\left.\quad\left(W-\frac{\kappa \chi}{r} \int_{0}^{t} \frac{\partial W}{\partial r} \mathrm{~d} t^{\prime}\right)\right|_{r=r_{0}}=0, \\
& W(\infty, t)=0, \quad W(r, 0)=0, \quad P_{w}=W\left(r_{0}, t\right) .
\end{aligned}
$$

Let the medium get the volume strain $\varepsilon$ at the end of the stage of long-term precursors. Assuming this moment as initial moment, we suppose that portion $m$ of strain $\varepsilon$ drops at the phase of short-term precursors after initial moment. We will assume this drop is instantaneous. Then we substitute function $e=-m \varepsilon E(t)$ into the right-hand side of differential equation from (11) or from (20).

Further we introduce dimensionless variables according to expressions

$$
w=\frac{\beta}{q m Q \sin 2 \psi} W, \quad \rho=\frac{r}{\sqrt{\kappa T}}, \quad \tau=\frac{t}{T}, \quad n=\frac{\sqrt{\kappa T}}{H}, \quad Q=\frac{\alpha s}{3 G} .
$$

If $T=86,400 \mathrm{~s}$ then the unit of dimensionless time corresponds to one day. At $\kappa=25 / 864 \mathrm{~m}^{2} / \mathrm{s}$ the unit of dimensionless distance will be $50 \mathrm{~m}$; the diffusing particle travels this distance in a day and $500 \mathrm{~m}$ in 100 days. The meaning of parameters $Q, n, \psi$ and the specific form of the right-hand side of the differential equation are given in Appendix A. As a result we obtain the following system

$$
\begin{aligned}
& \frac{\partial w}{\partial \tau}-\left(\frac{\partial^{2} w}{\partial \rho^{2}}+\frac{1}{\rho} \frac{\partial w}{\partial \rho}\right)=\delta(\tau) \theta(\tilde{\rho}, n, \rho),\left.\quad\left(w-\frac{\chi}{\rho} \int_{0}^{\tau} \frac{\partial w}{\partial \rho} \mathrm{d} \tau^{\prime}\right)\right|_{\rho=\rho_{0}}=0, \\
& w(\infty, \tau)=0, \quad w(\rho, 0)=0, \quad p_{w}(\tau)=w\left(\rho_{0}, \tau\right) .
\end{aligned}
$$

The solution of system (22) is calculated as follows. Let $u(\rho, \tau)$ be the solution of nonuniform equation and it satisfies two zero conditions: initial condition and condition at infinity. Then, this solution is expressed via the Duhamel integral which in this case takes the form

$$
u(\tilde{\rho}, \rho, \tau)=\frac{1}{2 \tau} e^{-\frac{\rho^{2}}{4 \tau}} \int_{0}^{\infty} z \theta(\tilde{\rho}, n, z) e^{-\frac{z^{2}}{4 \tau}} I_{0}\left(\frac{\rho z}{2 \tau}\right) \mathrm{d} z,
$$

where $I_{0}$ is the modified Bessel function.

Due to presence of delta-function, formula (23) actually expresses the solution of the uniform equation with 
nonzero initial condition but it is unimportant.

We take the Laplace transform to system (22) with respect to $\tau$ with parameter $p$. Designate the Laplace transform operator by $L$. We introduce an auxiliary function $f$ satisfying the uniform equation and zero conditions at the initial moment and at infinity. The Laplace transformation of $f$ is

$$
L f=C(p) K_{0}(\rho \sqrt{p}),
$$

where $K_{0}$ is Macdonald function and $C(p)$ is an arbitrary function.

Now, the function $v=f+u$ is constructing by choosing $C(p)$ such that $v$ satisfies the zero condition in the well, i.e. $v\left(\rho_{0}, \tau\right)=0$. Its Laplace transformation is

$$
L v(\rho, p)=L u(\rho, p)-\frac{L u\left(\rho_{0}, p\right)}{K_{0}\left(\rho_{0} \sqrt{p}\right)} K_{0}(\rho \sqrt{p}) .
$$

Finally, we take the solution of system (22) in the form $w=f+v$. This solution should satisfy only the boundary condition in the well of system (22) since other conditions are already fulfilled. As a result, the Laplace transform of pressure in the well is expressed by formula

$$
L w\left(\rho_{0}, p\right)=\frac{\chi \sqrt{p} K_{1}\left(\rho_{0} \sqrt{p}\right) L u\left(\rho_{0}, p\right)}{\rho_{0} p K_{0}\left(\rho_{0} \sqrt{p}\right)+\chi \sqrt{p} K_{1}\left(\rho_{0} \sqrt{p}\right)}+\frac{\chi \sqrt{p} K_{0}\left(\rho_{0} \sqrt{p}\right) L u_{\rho}\left(\rho_{0}, p\right)}{\rho_{0} p K_{0}\left(\rho_{0} \sqrt{p}\right)+\chi \sqrt{p} K_{1}\left(\rho_{0} \sqrt{p}\right)}
$$

where $L u_{\rho}\left(\rho_{0}, \tau\right)=\left.L \frac{\partial u(\rho, \tau)}{\partial \rho}\right|_{\rho=\rho_{0}}$.

Direct calculations and analysis conducted in the next section show that in calculations of precursory variations, we can neglect the second addend and accept

$$
L w\left(\rho_{0}, p\right) \cong \frac{\chi \sqrt{p} K_{1}\left(\rho_{0} \sqrt{p}\right)}{\rho_{0} p K_{0}\left(\rho_{0} \sqrt{p}\right)+\chi \sqrt{p} K_{1}\left(\rho_{0} \sqrt{p}\right)} \operatorname{Lu}\left(\rho_{0}, p\right) .
$$

The inverse transformation of (27) is described in Appendix B.

\section{Results, Discussion and Conclusions}

In this section we discuss only precursory level variations. The solution of this problem is given by expression (27). The original function is expressed as a convolution of two functions and it is reasonable first to analyze these functions.

Calculating function $u(\tilde{\rho}, \rho, \tau)$ given by expression (23), we obtain the result unexpected, on the first glance. In the case of strong earthquakes (starting from magnitude 5 - 5.5) $u(\tilde{\rho}, \rho, \tau)$, as a function of time, is almost constant during tens, hundreds, and thousands days (depending on the position of the well in the earthquake preparation zone) and is almost equal to its initial value. Namely $u(\tilde{\rho}, \rho, \tau) \approx \theta(\tilde{\rho}, n, \rho)$. It is caused that volumetric deformation is distributed in medium with small gradients which decrease with removal from an epicentre, decrease from increase in depth of focus and from growth magnitude. We remind that the value of precursory strain does not depend on magnitude and it does not exceed about $10^{-4}$. At the same time, the size of area in a neighbourhood of the well that actually determines the level variation is not connected with earthquake parameters. Let this size equal to $0.5 \mathrm{~km}$. Estimating the size of the near-field (epicentral) zone, where the volume strain distribution is most heterogeneous, as 4 average radius of heterogeneity, we obtain the values of 10 , 25 , and $65 \mathrm{~km}$ from magnitudes of 5,6 , and 7 , respectively [1]. The conclusion is evident.

If $u(\tilde{\rho}, \rho, \tau)$ is constant then we obtain from (27)

$$
L w\left(\rho_{0}, p\right)=\frac{\chi K_{1}\left(\rho_{0} \sqrt{p}\right)}{\sqrt{p}\left(\rho_{0} p K_{0}\left(\rho_{0} \sqrt{p}\right)+\chi \sqrt{p} K_{1}\left(\rho_{0} \sqrt{p}\right)\right)} \theta\left(\tilde{\rho}, n, \rho_{0}\right)=F(p) \theta\left(\tilde{\rho}, n, \rho_{0}\right)
$$

or after the inverse Laplace transform 


$$
w\left(\rho_{0}, \tau\right)=F(\tau) \theta\left(\tilde{\rho}, n, \rho_{0}\right) .
$$

here function $F(\tau)$ determines the time scale of the process of the short-term precursors. Function $F(\tau)$ presents in Figure 1. As seen from Figure 1, the level 3/4 reaches after 1 month and 9/10 after 2 months.

Now we can write the approximate formula of precursory variations in level of liquid in the well applicable for strong earthquakes in the form

$$
l \approx m \frac{q}{\beta \sigma g} \varepsilon(x, y, z) F(t) .
$$

There are no published examples of field observations that would make it possible to compare in detail theoretical results and empirical data. We will discuss only the following case. Work [3] give the plot of water level variations in the well at an epicentral distance of $30 \mathrm{~km}$ prior to the Przhevalsk earthquake of 1970 of magnitude 6.8 (Figure 2). Sudden transition to a short-term precursor was assumed in calculations. Actually so does not happen. Therefore we must introduce the conditional point of the beginning of a short-term precursor and corresponding coordinates $(l, t)$ as it is showed on Figure 2. We see good conformity of calculated data (Figure 1)

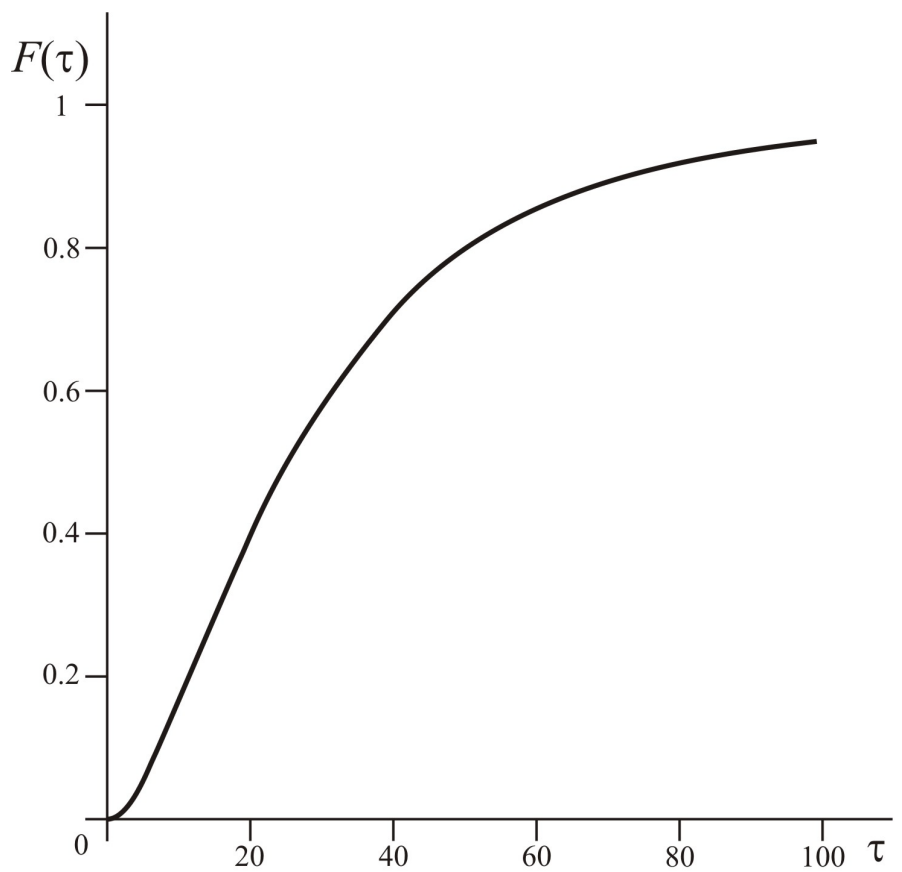

Figure 1. The plot of function $F(\tau)$. Unit of dimensionless time corresponds to one day.

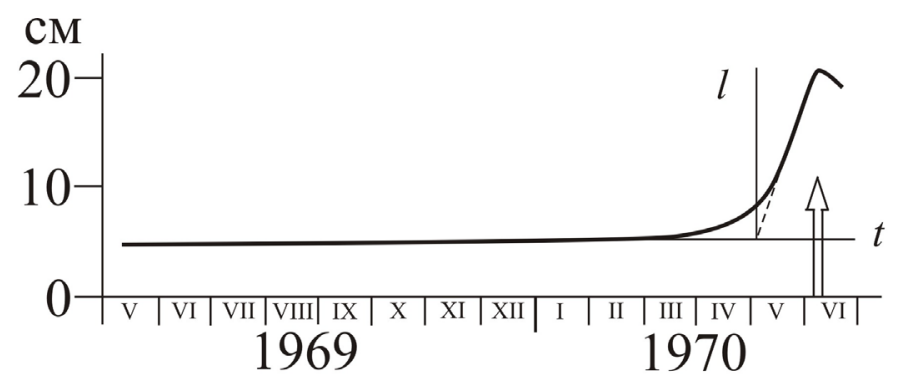

Figure 2. Plot of water level variations in the well before the Przevalsk earthquake of 1970. Figure is from [3]. The arrow is the moment of the earthquake. The origin of coordinates $(l, t)$ is the point of the conditional beginning of short-term precursor. 
and empirical data (Figure 2) in coordinates $(l, t)$. On these data it is impossible to predict the moment of earthquake. In general, comparison of Figure 1 and Figure 2 enables us to conclude that the theory well determines the time scale of the phenomenon. The precise value of level variation cannot be estimated: there are no data on earthquake epicenter location and the point of precursor in the system of tectonic stresses acting in the region (do this data on tectonic stresses exist anywhere?), there are no precise enough data on hypocenter depth, whereas the decrease in effect with depth is proportional to the cube of relative depth, etc. Thus, we can give only the general estimate. Assuming the earthquake was shallow and we accept: $m=5 \%, q=7, \beta=4 \times 10^{10}$ $\mathrm{Pa}^{-1}, s=10^{8} \mathrm{~Pa}, G=2 \times 10^{10} \mathrm{~Pa}, \alpha=0.1$. We obtain that the maximum level varies within $-50-+50 \mathrm{~cm}$ at the epicentral circle with a radius of $30 \mathrm{~km}$.

It is necessary to note the definite uncertainty in description of properties of medium. Times of action of Earth tides and precursors differ by two orders of magnitude; maximum values of strains differ by 3 - 4 orders of value; the medium is strongly affected during an earthquake. How do the properties of medium change in these conditions?

Despite of it we confirm that the existing theory of preparation of a tectonic earthquake and methods developed in the paper enables the effective calculation of variations in level of liquid in wells in the case of processes connected with tectonic earthquakes. This result is directly related with prediction problem of earthquake.

\section{References}

[1] Dobrovolsky, I.P. (2000) Theory of the Tectonic Earthquake Preparation. UIPE, Russian Academy of Sciences, Moscow, 134 p.

[2] Rikitake, T. (1975) Earthquake Prediction. ESPC, 374 p.

[3] Sadovsky, M.A., Nersesov, I.L., Nigmatullaev, S.K., et al. (1972) The Processes Preceding Strong Earthquakes in Some Regions of Middle Asia. Tectonophysics, 14, 295-307. http://dx.doi.org/10.1016/0040-1951(72)90078-9

[4] Kissin, I.G., Belikov, V.M. and Ishankuliev, G.A. (1990) Extreme Groundwater Table Fluctuations in a Seismically Active Region. Doklady AN SSSR, 314, 1099-1103.

[5] Dobrovolsky, I.P., Zubkov, S.I. and Miachkin, V.I. (1979) Estimation of the Size of Earthquake Preparation Zones. Pageoph, 117, 1025-1044. http://dx.doi.org/10.1007/BF00876083

[6] Doetsch, G. (1956) Anletung Zum Praktiscen Gebrauch Der Laplace_-Transformation. München, 208 p.

[7] Watson, G. (1945) A Treatise on the Theory of Bessel Functions. Cambridge, 1, 243 p. 


\section{Main Notations}

$t$ is time, [s];

$r$ and $\varphi$ are local polar coordinates with the origin at the well axis;

$h$ is thickness of water-saturated layer, [m];

$r_{0}$ is well radius, [m];

$l$ is variation in level of fluid in the well, [m];

$\sigma$ is density of the porous fluid, $\left[\mathrm{kg} / \mathrm{m}^{3}\right]$;

$g$ is gravity acceleration, $\left[\mathrm{m} / \mathrm{s}^{2}\right]$;

$H$ is depth of hypocenter of the spherical heterogeneity, [m];

$R$ is radius of the spherical heterogeneity, [m];

$G$ is shear modulus of the Earth's crust, $[\mathrm{Pa}]=\left[\mathrm{kg} /\left(\mathrm{m} \cdot \mathrm{s}^{2}\right)\right]$;

$s$ is tangential stress at infinity, [Pa];

$\alpha$ is relative increment in shear modulus in heterogeneity;

$e$ is the volume strain of elementary volume of porous two-phase medium;

$e_{m}$ is the volume strain of pores filled with fluid;

$q$ is coefficient in expression $e_{m}=q e$;

$k$ is permeability, $\left[\mathrm{m}^{2}\right]$;

$\mu$ is coefficient of dynamical viscosity of fluid, [Pa.s];

$m$ and $m_{0}$ are the current and initial porosity of the medium;

$\beta$ is isothermal compressibility of fluid, $\left[\mathrm{Pa}^{-1}\right]$;

$E$ is Heaviside step function;

$\delta$ is delta function;

$\Delta$ is Laplace operator with respect to spatial variables;

$i$ is imaginary unit.

\section{Appendix A. Volume Strain}

The cycle of tectonic earthquake consists in beginning, development and disintegration of heterogeneity of properties in the Earth's crust [1] [5]. We introduce the Cartesian system of coordinates $x, y, z$ in a half-space $z \geq 0$ with its origin at the heterogeneity epicenter and coordinate axes orient along tangential stresses $s$ at infinity. To the beginning of acting of short-term precursors, the medium gets the volume strain [1]

$$
\varepsilon=\frac{\alpha s R^{3}}{3 G} 2 x y\left(\frac{1}{r_{1}^{5}}+\frac{2}{r_{2}^{5}}-\frac{10 H(z+H)}{r_{2}^{7}}+R^{2}\left[\frac{14(z+H)^{2}}{r_{2}^{9}}-\frac{2}{r_{2}^{7}}\right]\right),
$$

where $r_{1}=\sqrt{x^{2}+y^{2}+(z-H)^{2}}$ and $r_{2}=\sqrt{x^{2}+y^{2}+(z+H)^{2}}$.

Now we introduce dimensionless variables and polar coordinates $(\tilde{\rho}, \psi)$ by expressions

$$
(\xi, \eta, \zeta)=\frac{(x, y, z)}{H}, \quad \tilde{\rho}=\sqrt{\xi^{2}+\eta^{2}}, \quad \xi=\tilde{\rho} \cos \psi, \quad \eta=\tilde{\rho} \sin \psi .
$$

Besides, we consider the near-surface layer and, thus, assume $z=0$; this case is typical for wells and shallow boreholes. The earthquake considered is also shallow, so $R=H$, and the effect will be maximum. Then

$$
\varepsilon=Q \theta_{0} \sin 2 \psi=Q \tilde{\rho}^{2}\left(\frac{3}{Z^{5}}-\frac{12}{Z^{7}}+\frac{14}{Z^{9}}\right) \sin 2 \psi,
$$

where $Z=\sqrt{\tilde{\rho}^{2}+1}, Q=\frac{\alpha s}{3 G}$ and notation $\theta_{0}$ is obvious.

Evidently, $\varepsilon$ is the product of two functions depending on angle and radius; the coordinate axes are zero lines.

Further it is investigated the filtration problem in the well vicinity.

Let the well axis is located at point $x, y$. We place the coordinate origin of the filtration problem at this point. Then $x$ and $y$ are parameters and variables will be $r, \varphi$. Then in (A.1) coordinates, considering 
(A.2) and (21), transform by the following scheme (for example, for $x$ )

$$
\frac{x}{H} \Rightarrow \frac{x+r \cos \varphi}{H}=\xi+\frac{\sqrt{\kappa T}}{H} \rho \cos \varphi=\xi+n \rho \cos \varphi .
$$

here the scale ratio $n=\sqrt{\kappa T} / H$ is introduced automatically.

Now after average $\varepsilon$ on angle $\varphi$ we receive

$$
\bar{\varepsilon}=Q \sin 2 \psi \theta(\tilde{\rho}, n, \rho) .
$$

Function $\theta(\tilde{\rho}, n, \rho)$ is complicated. It involves $\boldsymbol{K}$ and $\boldsymbol{E}$, which are the complete elliptic integrals of the first and second kinds accordingly, and consists 4 component

$$
\begin{gathered}
\theta(\tilde{\rho}, n, \rho)=S_{1}+S_{2}+S_{3}+S_{4}, \\
S_{1}=B \lambda^{2}(1-\lambda)\left(150(\lambda-1)^{2} b^{4}-104 \lambda(\lambda-1)(\lambda-2) b^{2}+\lambda^{2}\left(24 \lambda^{2}-71 \lambda+71\right)\right) \boldsymbol{K}(\sqrt{\lambda}) \\
S_{2}=-2 B \lambda\left(90(\lambda-1)^{3} b^{6}-150 \lambda(\lambda-2)(\lambda-1)^{2} b^{4}\right. \\
\left.+13 \lambda^{2}(\lambda-1)\left(8 \lambda^{2}-23 \lambda+23\right) b^{2}-4 \lambda^{3}(\lambda-2)\left(6 \lambda^{2}-11 \lambda+11\right)\right) \boldsymbol{E}(\sqrt{\lambda}), \\
S_{3}=12 n^{2} \rho^{2}(\lambda-1) B\left(40(\lambda-1)^{3} b^{4}-8 \lambda\left(\lambda^{2}-1\right)(\lambda-2)(2 \lambda-1) b^{2}\right. \\
\left.+\lambda^{2}\left(8 \lambda^{4}-21 \lambda^{3}+17 \lambda^{2}+8 \lambda-4\right)\right) \boldsymbol{K} \\
S_{4}=-24 n^{2} \rho^{2} B\left(10(\lambda-2)(\lambda-1)^{3} b^{4}-2 \lambda(\lambda-1)\left(8 \lambda^{4}-19 \lambda^{3}+15 \lambda^{2}+8 \lambda-4\right) b^{2}\right. \\
\left.+\lambda^{2}(\lambda-2)\left(8 \lambda^{4}-12 \lambda^{3}+11 \lambda^{2}+2 \lambda-1\right)\right) \boldsymbol{E}(\sqrt{\lambda}),
\end{gathered}
$$

where $B=\frac{\sqrt{2 \lambda}}{120 \pi b^{9}(1-\lambda)^{4}}, \lambda=\frac{4 \tilde{\rho} n \rho}{1+(\tilde{\rho}+n \rho)^{4}}, \quad b=\sqrt{2 \tilde{\rho} n \rho}$ and at $\rho \rightarrow \infty$ or $\tilde{\rho} \rightarrow \infty \quad \theta \rightarrow 0$.

The important remark is: obviously, it is possible to choose any other process of deformation of medium, but the technique will be the same.

\section{Appendix B. Inverse Laplace Transformation}

The inverse Laplace transformation is defined by the expression [6]

$$
F(t)=\frac{1}{2 \pi i} \int_{c-i \infty}^{c+i \infty} f(p) e^{p t} \mathrm{~d} p
$$

where $c \geq c_{0}$ and $c_{0}$ is the convergence abscissa of the direct transformation.

Now possible direct application of the formula (B.1), because existing computer software enables calculation of known, including special, functions in the whole complex area of their determination. Usually, such evaluations give numerical values in isolated points. It is convenient for check of a correctness of other methods.

We will act usually and select the most convenient path of integration amongst the set of possible paths in the area, where the function under integral is analytical. Surely, this way does not reduce the attractiveness of analytical solution that is still preferable.

Apparently from (28) and (29), we should find the original from

$$
F(p)=\frac{\sqrt{p} K_{1}(\sqrt{p})}{p K_{0}(\sqrt{p})+\chi \sqrt{p} K_{1}(\sqrt{p})} .
$$

Bessel functions are single-valued in complex plane with a branch cut along the negative semi-axis [7]. This property is also valid for function $F(p)$. Then two paths of integration are really interesting: 1 ) along the imaginary axis and a positive semicircle centered at the origin of coordinates, 2) along two sides of the negative 
semi-axis. The second path is easier and the inversion expression for it after several transformations is

$$
-\frac{2}{\pi} \int_{0}^{\infty} z \operatorname{Im} F\left(-z^{2}\right) e^{-z^{2} t} \mathrm{~d} z
$$

where integral is taken from the imaginary part.

It is reasonable to represent function $F(p)$ as the sum

$$
F(p)=\frac{1}{\sqrt{p}}+\frac{\sqrt{p} K_{1}(\sqrt{p})-\sqrt{p} K_{0}(\sqrt{p})-\chi K_{1}(\sqrt{p})}{p K_{0}(\sqrt{p})+\chi \sqrt{p} K_{1}(\sqrt{p})} .
$$

here the first term has the original $1 / \sqrt{\pi t}$ and the second term has no singularities. 